\title{
Acknowledgement to Reviewers of Foods in 2015
}

\author{
Foods Editorial Office \\ Published: 22 January 2016 \\ MDPI AG, Klybeckstrasse 64, CH-4057 Basel, Switzerland; foods@mdpi.com
}

The editors of Foods would like to express their sincere gratitude to the following reviewers for assessing manuscripts in 2015.

We greatly appreciate the contribution of expert reviewers, which is crucial to the journal's editorial decision-making process. Several steps have been taken in 2015 to thank and acknowledge reviewers. Good, timely reviews are rewarded with a discount off their next MDPI publication. By creating an account on the submission system, reviewers can access details of their past reviews, see the comments of other reviewers, and download a letter of acknowledgement for their records. In addition, MDPI has launched a collaboration with Publons, a website that seeks to publicly acknowledge reviewers on a per journal basis. This is all done, of course, within the constraints of reviewer confidentiality. Feedback from reviewers shows that most see their task as a voluntary and mostly unseen work in service to the scientific community. We are grateful to our reviewers for the contribution they make.

Aguedo, Mario

Alavi, Sajid

Allegaert, Karel

Aluko, Rotimi

Arvanitoyannis, Ioannis

Aubourg, Santiago P.

Barba, Francisco J.

Beasley, Shea

Beatty, Stephen

Bermúdez-Aguirre, Daniela

Bianco, Ismael D.

Bidwell, Joseph

Blanco Penedo, Isabel

Bourquin, Leslie

Britten, Michel

Burrows, Tracy L.

Canavari, Maurizio

Cano, Amalia

Caporaso, Nicola

Cardoso, Marisa

Casal, Susana

Castro, Sonia
Cavazza, Antonella

Chang, Ching-Jui

Chang, Fang-Rong

Chaves-Lopez, Clemencia

Chen, Huaiqiong

Chiang, Wen-Dee

Ciani, Maurizio

Das, Undurti

Donno, Dario

Dreij, Kristian

Drosinos, Eleftherios H.

Durango, Diego L.

Durazzo, Alessandra

Economou, Vangelis

Edwards, Sandra

Elansary, Hosam O.

Elkhalifa, Abd Elmoneim O.

Everett, David W.

Fabra Rovira, M. Jose

Fåk, Frida

Feigl, Beatrix

Ferragut, Victoria
Fitton, Helen

Fjeld, Heidi

Fletcher, Paul C.

Fontecha, Javier

Fortunati, Elena

Foster, Jane A.

Fraqueza, Maria João

Frígola, Ana

GonzÁlez-DÍaz, Humberto

González-Mas, M. Carmen

Greco, Luigi

Gruszecki, Wieslaw

Guerzoni, Maria Elisabetta

Hallmann, Ewelina

Hamdy Roby, Mohamed Hussein

Hansen, Tina Beck

Harrold, Joanne A.

Hasanuzzaman, Mirza

Henriques, Marta H. F.

Holck, Askild Lorentz

Holley, Richard A.

Hönow, Ruth 
Hospital, Xavier F.

Hsu, Chia-Lin

Iametti, Stefania

Ibrahim, Salam A.

Iossifidou, Eleni

Jahncke, Michael

Jensen, Morten Georg

Johansson, Eva

Johnson-Kozlow, Marilyn

Jordan, Kieran

Joseph, Lucy

Juneja, Vijay

Juszczak, Lesław

Kasarda, Don

Khachik, Fred

Kimiya, Takashi

Kjarnes, Unni

Knight, John

Kojo, Shosuke

Komaitis, Michael

Koppel, Kadri

Kourkoutas, Yiannis

Kovbasnjuk, Olga

Lalas, Stavros

Lamuela Raventós, Rosa María

Langton, Maud

Larre, Colette

Leone, Antonella

Lillioja, Stephen

Lo Scalzo, Roberto

Longo, Vincenzo

Loveday, Simon M.

Maher, Pamela

Maheshwari, Akhil

Marchello, John

Marín, Sonia

Martinez-Monteagudo, Sergio
Masson, Lourdes M. P.

Mataragas, Marios

Mattei, Josiemer

Miller, Charles

Miyagi, Atsuko

Molenaar, Jaap

Morabito, Antonino

Moreira , Ramón

Moreno, Silvia

Morita, Kyoji

$\mathrm{Mu}$, Jianhong E.

Muñoz-Alférez, M. José

Myers, James

Nag, Arup

Nemeghaire, Stéphanie

Newnham, Evan

Nicolai, Taco

Nicoli, Maria Cristina

Niewold, Theo

Nolan, John

Novellino, Ettore

Omer, Sayed

Panagou, Efstathios

Park, Chung Gyoo

Parsons, Barry J.

Pérez-Lamela, Concepción

Perrone, Serafina

Piga, Antonio

Plourde, Guy

Prakash, Anuradha

Puppo, Cecilia

Rajagopal, S. N.

Rallabhandi, Prasad

Rembialkowska, Ewa

Rivas, Ana

Rivero-Pérez, M. Dolores

Robitaille, Gilles
Rocha Guzmán, Nuria Elizabeth

Rodriguez-Mateos, Ana M.

Rosa Loizzo, Monica

Rumpold, Birgit

Salafranca Lázaro, Francisco J.

Santillo, Antonella

Santini, Antonello

Scherf, Katharina

Sgroi, Filippo

Sheikh, Ali Ahmad

Silva, Juan L.

Smith, Brennan

Sobarzo-Sánchez, Eduardo

Soultos, Nikolaos

Speer, Karl

$\mathrm{Su}$, Marcia Shu-Wei

Syed, Moinuddin

Talens, Pau

Tarantino, Giovanni

Tassou, Chrysoula C.

Topp, Kairsty

Tormod, Mørk

Torrieri, Elena

Tzia, Constantina

Ueno, Hideto

Velickova, Elena

Verhagen, Hans

Vermeer, Herman Maarten

Erdman, John W.

Wallace, R. John

Wang, Chung-Yi

West, Gale

Wu, Chi-Rei

Xin, Xiu-Fang

Yamamoto, Kazutaka

Ying, Danyang

Zhu, Yefei 